\title{
Circular RNA hsa_circ_0075828 promotes bladder cancer cell proliferation through activation of CREB1
}

\author{
Chengle Zhuang ${ }^{1}$, Xinbo Huang ${ }^{1}$, Jing $\mathrm{Yu}^{2}{ }^{2}$ * $\mathcal{E}$ Yaoting $\mathrm{Gui}^{1, *}$ \\ ${ }^{1}$ Guangdong and Shenzhen Key Laboratory of Male Reproductive Medicine and Genetics, Institute of Urology, Peking University Shenzhen \\ Hospital, Shenzhen-Peking University-the Hong Kong University of Science and Technology Medical Center, Shenzhen 518000, \\ ${ }^{2}$ Department of Laboratory Medicine, Peking University Shenzhen Hospital, Shenzhen 518000, PR China
}

\begin{abstract}
Circular RNAs (circRNAs), one kind of non-coding RNA, have been reported as critical regulators for modulating gene expression in cancer. In this study, microarray analysis was used to screen circRNA expression profiles of bladder cancer (BC) 5637 cells, T24 cells and normal control SV-HUC-1 cells. The data from the microarray showed that hsa_circ_0075828 (named circCASC15) was most highly expressed in 5637 and T24 cells. circCASC15 was highly expressed in BC tissues and cells. Overexpression of circCASC15 was closely associated with BC tumor stage and promoted cell proliferation significantly in vitro and in vivo. Mechanistically, circCASC15 could act as miR-1224-5p sponge to activate the expression of CREB1 to promote cell proliferation in BC. In short, circCASC15 promotes cell proliferation in $\mathrm{BC}$, which might be a new molecular target for $\mathrm{BC}$ diagnosis and therapy. [BMB Reports 2020; 53(2): 82-87]
\end{abstract}

\section{INTRODUCTION}

Bladder cancer (BC) is one of the most common malignancies of the urinary system, with high morbidity and mortality rates worldwide (1). Surgery, radiation and chemotherapy are effective therapies for a subset of BC patients (2). However, the overall therapeutic efficacy and the 5-year survival rate are still low (3). In addition, the molecular mechanisms of BC pathogenesis are poorly understood (4). Thus, discovering novel molecular biomarkers and further investigation of the mechanisms of $\mathrm{BC}$ progression are important.

*Corresponding authors. Yaoting Gui, Tel: +86-755-839233; Fax: +86-755-839233; E-mail: guiyaoting2007@aliyun.com; Jing Yu, Tel: +86-755-839233; Fax: +86-755-839233; E-mail: jing_yu2004@ aliyun.com

https://doi.org/10.5483/BMBRep.2020.53.2.059

Received 25 February 2019, Revised 27 March 2019, Accepted 3 May 2019

Keywords: Bladder cancer, circCASC15, Circular RNA, CREB1, miR-1224-5p
Circular RNAs (circRNAs), a class of non-coding RNAs, are closed covalent loops with neither a polyadenylate tail nor $5^{\prime}$ to $3^{\prime}$ polarity (5). More than 30,000 circRNAs have been identified, and they are stable, conserved and abundant in mammalian cells (6). Various circRNAs have been suggested to be involved in the occurrence of numerous tumors, including gastric cancer, hepatocellular carcinoma, prostate cancer, and BC (7). Numerous studies have reported that miRNA response elements (MREs) exist in circRNAs, which function as competing endogenous RNAs (ceRNAs) to sponge miRNAs or transcription regulators to regulate the expression of target genes (8). This hypothesis was first verified by solid evidence that there are 70 miR-7 binding sites in circRNA CiRS-7 that act as miR-7 sponges to regulate miR-7 target mRNA expression (8). Recently, circular RNA YAP1 has been shown to directly interact with miR-367-5p to modulate $p 27$ expression and inhibit proliferation and invasion of gastric cancer (9). A study reported that circFBLIM1 promoted hepatocellular carcinoma through sponging miR-346 (10). Circular RNA cTFRC sponged miR-107 to increase TFRC expression to promote BC progression (11). Circular RNA circPRMT5 acted as a miR-30c sponge to induce epithelialmesenchymal transition (EMT) in BC (12). Circular RNA circHIPK3 bound to miR-558 to suppress heparanase expression in BC (13). In brief, the circRNA/miRNA/mRNA axis may be extensively involved in the progression of cancer, including $\mathrm{BC}$, and the underlying mechanisms of circRNAs are still not clear.

In this study, we mainly focus on a circRNA derived from CASC15 gene, hsa_circ_0075828, termed circCASC15, which was screened from microarray analysis. The expression of circCASC15 was increased significantly in BC tissues and notably associated with the tumor stage of BC. Additionally, the high abundance of circCASC15 promoted proliferation of BC through acting as a ceRNA for miR-1224-5p. It then activated oncogenic cAMP responsive element binding protein 1 (CREB1) expression in BC. Collectively, circCASC15 may be a novel potential target for the treatment of $\mathrm{BC}$. 


\section{RESULTS}

The expression of circCASC15 was upregulated in BC tissues and cell lines and was mainly located in cytoplasm

In this study, we screened circRNA expression in BC 5637, T24 and SV-HUC-1 cells. We normalized the intensity of these three cell lines (Fig. S1A), drew scatter diagrams (5637 vs SV-HUC-1 and T24 vs SV-HUC-1) (Fig. S1B and C) and showed the corresponding heatmap (Fig. S1D and E). Compared with SV-HUC-1, circCASC15 (also named hsa_circ_0075828 in circBase database) was the most highly expressed in 5637 and T24 (Table S1). So we picked this circRNA for further study. As presented in Fig. 1A, the circCASC15, 204 nucleotides in length, was generated from CASC15 gene. Divergent primers for circCASC15 were designed and used to obtain circular product with splice junction, and the product was confirmed by Sanger sequencing. The circCASC15 expression level was increased significantly in 41 of 67 pair normal and BC tumor tissues (Fig. 1B). The expression of circCASC15 in tumors was approximately 3 times as high as that in para-cancer tissues (Fig. 1C, P $<0.01$ ). Compared with SV-HUC-1 cells, the expression of circCASC15 was upregulated significantly in 5637, T24, J82, UMUC-3 and SW780 cells (Fig. 1D). Owing

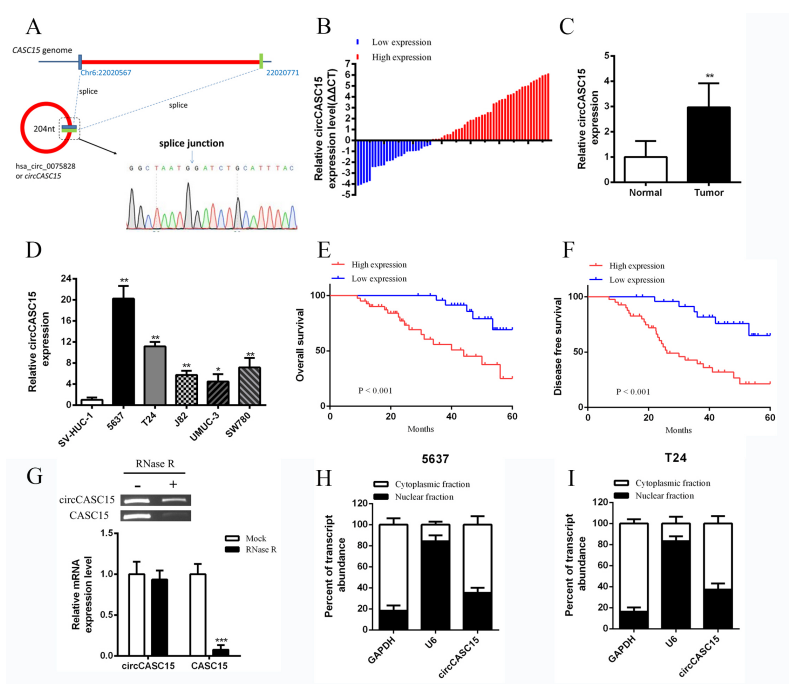

Fig. 1. The expression characteristics of circCASC15, its correlation with prognosis and location in BC are presented. (A) The schematic diagram represents the genomic location and splicing pattern of circCASC15. Direct sequencing was used to verify the splice junction. (B) The expression pattern of circCASC15 in 67 pairs of BC tissues was shown. (C, D) circCASC15 was significantly upregulated in BC tissues and cells. (E, F) High circCASC15 expression indicated poor overall and disease free survival of $\mathrm{BC}$ patients. (G) circCASC15 was resistent to RNase $\mathrm{R}$. $(\mathrm{H}, \mathrm{I})$ circCASC15 was mainly located in the cytoplasm of BC 5637 and T24 cells. $* \mathrm{P}<0.05, * * \mathrm{P}<0.01$ and $* * * P<0.001$. to the high abundance of circCASC15 in 5637 and T24 cell lines compared with the other three $\mathrm{BC}$ cell lines, we choose 5637 and T24 cells for further experiments. Next, the correlation between circCASC15 expression and the clinicopathological characteristics of BC patients was determined. As shown in Table S2, there was no relationship among age, gender, tumor size, lymph modes metastasis and expression levels of circCASC15. Nonetheless, tumor stage had a significant association with highly expressed circCASC15 $(P=0.011)$. Besides, high expression of circCASC15 indicated low overall and disease free survival (Fig. $1 \mathrm{E}$ and $\mathrm{F}$, both $\mathrm{P}<0.001$ ). Exonuclease RNase $\mathrm{R}$ was used to validate the circular form of circCASC15 and results illustrated that circCASC15 was resistent to RNase R (Fig. 1G). Finally, we found that circCASC 15 was mainly localized in the cytoplasm of BC 5637 and T24 cells (Fig. $1 \mathrm{H}$ and I). These results indicated that the expression of circCASC15 was upregulated in BC tissues and cells. Highly expressed circCASC15 was associated closely with BC tumor stage. In addition, circCASC15 mainly existed in the cell cytoplasm.

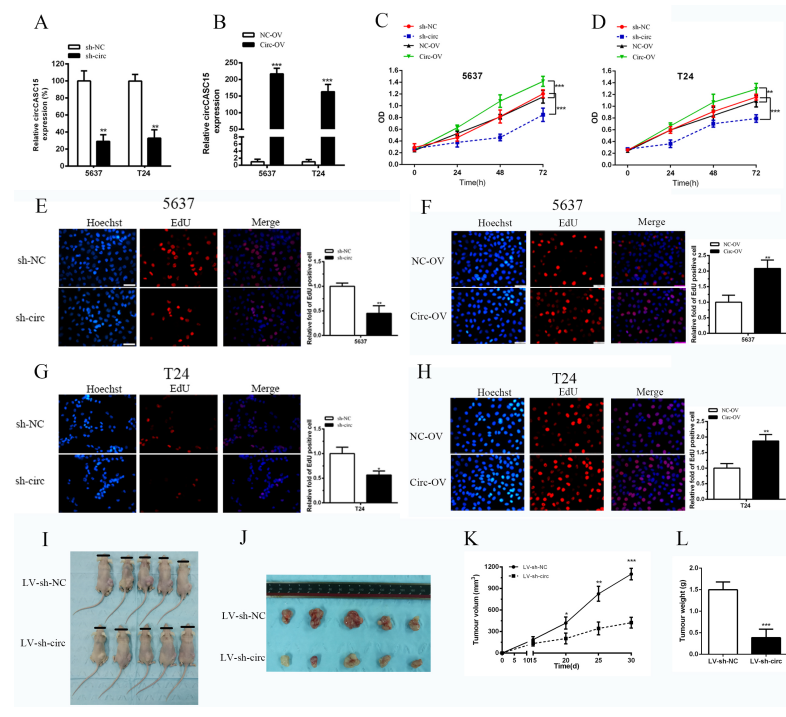

Fig. 2. circCASC15 promotes $B C$ cell proliferation significantly in vitro and in vivo. (A, B) The expression level of circCASC15 was decreased or increased significantly after knockdown or overexpression of circCASC15 in 5637 and T24 cells, respectively. (C, D) Cell proliferation was inhibited or promoted significantly after knockdown or overexpression of circCASC15 in 5637 and T24 cells using the CCK-8 assay, respectively. (E-H) Cell growth was suppressed or activated remarkably after knockdown or overexpression of circCASC15 in 5637 and T24 cells using the EdU assay, respectively (bar in EdU image represents $50 \mu \mathrm{m}$ ). (I, J) Representative images of the tumor in nude mice were shown. $(\mathrm{K}, \mathrm{L})$ Compared with the LV-sh-NC group, the tumor volume curve and tumor weight were significantly inhibited in the LV-sh-circ group. $* \mathrm{P}<0.05, * * \mathrm{P}<0.01$ and $* * * \mathrm{P}<0.001$. 


\section{Downregulation of circCASC15 inhibited proliferation in BC in vitro and in vivo}

Compared with the corresponding controls, the results from qRT-PCR showed that the expression level of circCASC15 was decreased or increased significantly after transfection of shRNA targeting the circCASC15 plasmid (sh-circ) or overexpression of the circCASC15 plasmid (Circ-OV), respectively (Fig. 2A and B). After knockdown or overexpression of circCASC15 in BC cells, cell proliferation was suppressed or promoted remarkably in the 5637 and T24 cell lines, respectively (Fig. 2C and D). To verify the effects of circCASC15 on cell growth, we performed another cell proliferation assay, the $\mathrm{EdU}$ assay, to measure cell proliferation. As presented in Fig. 2E and F, EdU positive cells were decreased or increased significantly after knockdown or overexpression of circCASC15 in 5637, respectively. Similarly, cell growth was suppressed or promoted remarkably after knockdown or overexpression of circCASC15 in T24, respectively (Fig. 2G and $\mathrm{H}$ ). However, circCASC15 has no effects on cell migration, invasion and apoptosis in BC 5637 and T24 cells (data was not presented). In addition, 5637 cells stably transfected with sh-circ or sh-NC were injected into nude mice. Thirty days later, mice were sacrificed, and tumors were harvested (Fig. 2I). The tumor volume in LV-sh-circ was remarkably smaller than that in LV-sh-NC group (Fig. 2J). As presented in Fig. 2K, the tumor volume curve was significantly suppressed in LV-sh-circ group. In addition, tumor weight in LV-sh-circ group was obviously less than in the LV-sh-NC group (Fig. 2L). However, the mice body weight was no difference between LV-sh-NC and LV-sh-circ during 30 days of feeding (Fig. S2). In brief, circCASC15 promotes proliferation in $\mathrm{BC}$ in vitro and in vivo.

\section{circCASC15 directly bound to miR-1224-5p and CREB1 was a target of miR-1224-5p}

Studies have shown that circRNAs may function as sponges to bind to miRNAs when mainly located in cytoplasm (14). In this study, circCASC15 was mainly localized in BC cytoplasm, and bioinformatics analysis (15) suggested that circCASC15 may bind to five miRNAs (Fig. 3A). Evidences have proven that miRNAs degrade mRNA and inhibit translation of their targets in an AGO2-dependent manner (16). Thus, anti-AGO2 immunoprecipitation (RIP) was performed in 5637 cells transfected with each miRNA mimics to pull down circCASC15 utilizing anti-IgG (control) or anti-AGO2 antibodies, followed by qRT-PCR. The results indicated that the expression level of circCASC15 bound to anti-AGO2 antibodies was increased remarkably in cells transfected with miR-1224-5p mimics compared to the control, with the except of the other four miRNA mimics (Fig. 3B). The RT-PCR results revealed that when we used anti-AGO2 antibodies for RIP, circCASC15 was enriched significantly in cells transfected with miR-1224-5p mimics compared with the control (Fig. 3C). These results suggested that miR-1224-5p could directly target circCASC15 in an AGO2-dependent manner. In addition, the wide type circCASC15 (WT) with binding sites of miR-1224-5p or a mutated circCASC15 (Mut) sequence was inserted into 3' UTR of renilla luciferase in the psiCHECK-2 vector (Fig. S3A). The results showed that the luciferase activities of WT reporter were decreased or increased significantly after overexpression or inhibition of miR-1224-5p, respectively, in 5637 and T24 cells (Fig. S3B and C). However, no difference was shown in the mutated luciferase reporter. These results implied that circCASC15 may bind to miR-1224-5p directly and function as a ceRNA for miR-1224-5p. According to the prediction from TargetScan databases (http://www.targetscan.org) (17), miR1224-5p could target CREB1 with a high score. The wide type CREB1-3' UTR (WT) with binding sites of miR-1224-5p or mutated CREB1 (Mut) sequence was inserted into the $3^{\prime}$ UTR of renilla luciferase in the psiCHECK-2 vector (Fig. S3D). The results suggested that the luciferase activities were suppressed significantly after transfection of miR-1224-5p mimics in the CREB1 3' UTR WT group compared with miR-NC in 5637 and T24 cells (Fig. S3E and F). Conversely, transfection of miR-1224-5p inhibitor could remarkably increase the luciferase activities in the WT group compared with NC inhibitor in 5637 and T24 cells. No significance was shown in the CREB1 Mut group in 5637 and T24 cells. Furthermore, the

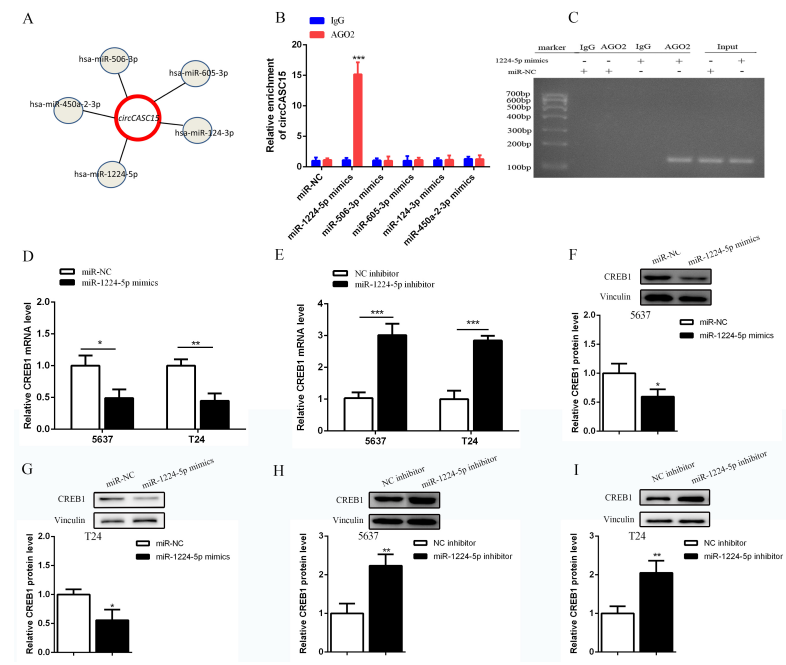

Fig. 3. circCASC15 directly binds to miR-1224-5p that targets downstream CREB1 in BC. (A) Bioinformatics analysis showed five possible miRNAs targeting circCASC15. (B, C) RIP assay demonstrated that circCASC15 bound to miR-1224-5p directly. (D, E) The mRNA expression level of CREB1 was decreased or increased significantly after overexpression or inhibition of miR-1224-5p in 5637 and T24 cells, respectively. $(\mathrm{F}, \mathrm{G})$ The CREB1 protein expression level was inhibited remarkably after transfection of miR-1224-5p mimics in 5637 and T24 cells. $(\mathrm{H}, \mathrm{l})$ The CREB1 protein expression level was increased notably after transfection of miR-1224-5p inhibitors in 5637 and T24 cells. ${ }^{* P}<0.05, * * P<0.01$ and $* * * P<0.001$ 
CREB1 mRNA level was decreased or increased significantly after overexpression or inhibition of miR-1224-5p in 5637 and T24 cells, respectively (Fig. 3D and E). Similarly, the CREB1 protein level was suppressed markedly after transfection of miR-1224-5p mimics in 5637 (Fig. 3F) and T24 (Fig. 3G) cells, whereas the miR-1224-5p inhibitor exhibited an opposite role in 5637 (Fig. 3H) and T24 (Fig. 3l) cells. In short, these data indicated that CREB1 was the target of miR-1224-5p.

\section{CREB1 was the functional target of circCASC15}

To investigate whether the high abundance of circCASC15 promoted cell proliferation through activating the expression of CREB1, the rescue assay was performed. We found that CREB1 mRNA expression level was decreased significantly in 5637 and T24 cells after knockdown of circCASC15 (Fig. 4A and $B)$. However, the reduction effects could be abrogated by overexpression of CREB1. Western blot indicated that downregulation of circCASC15 remarkably suppressed CREB1 protein expression levels in 5637 and T24 cells, whereas the inhibitory effects could be abolished by upregulation of CREB1 (Fig. 4C and D). In addition, as presented in Fig. 4E-H, CCK-8 and EdU data revealed that downregulation of circCASC15 inhibited cell proliferation significantly in 5637 and T24 cells.

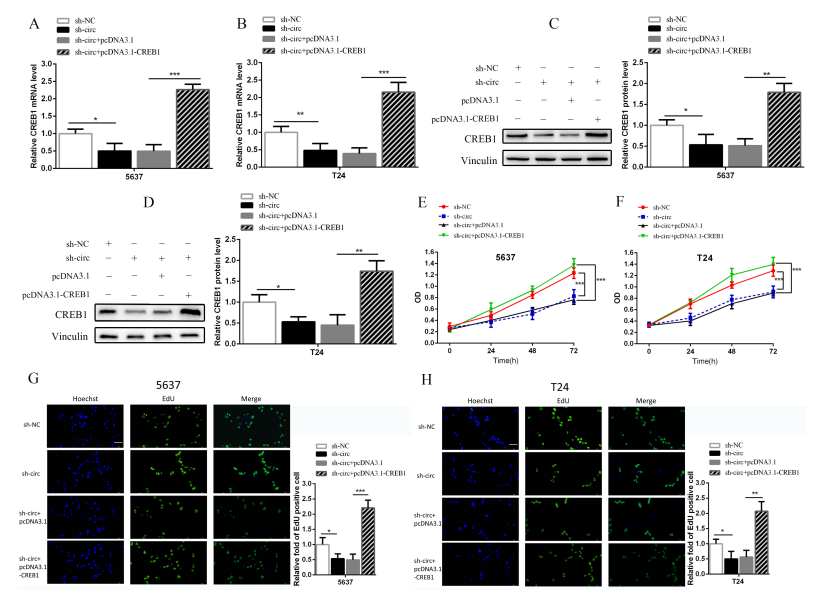

Fig. 4. CREB 1 was the functional target of circCASC15. (A, B) The CREB1 mRNA expression level was inhibited significantly after knockdown of circCASC15 in 5637 and T24 cells. However, overexpression of CREB1 could significantly abrogate the inhibitory effects of knockdown of circCASC15 on CREB1 mRNA expression level in 5637 and T24 cells. (C, D) The CREB1 protein expression level was suppressed remarkably after downregulation of circCASC15 in 5637 and T24 cells. But overexpression of CREB1 could notably counteract the inhibitory effects of knockdown of circCASC15 on CREB1 protein expression level in 5637 and T24 cells. (E-H) Cell proliferation was inhibited significantly after inhibition of circCASC15 in BC 5637 and T24 cells using CCK-8 and EdU assays. However, overexpression of CREB1 could markedly abolish the suppressive effects of knockdown of circCASC15 on cell growth (bar in EdU image represents $50 \mu \mathrm{m})$. ${ }^{*} \mathrm{P}<0.05, * * \mathrm{P}<0.01$ and $* * * \mathrm{P}<0.001$.
Nonetheless, cotransfection of CREB1 and sh-circ could counteract the suppression effects on cell growth in BC 5637 and T24 cells. These results demonstrated that circCASC15 promoted cell proliferation partly via activating CREB1 expression, and CREB1 was the functional target of circCASC 15.

\section{DISCUSSION}

Various studies have shown that many circRNAs in the cytoplasm play oncogenic or tumor-suppressive roles in distinct human cancers through functioning as miRNA sponges (18). CircRNA circ-ITCH sponged miR-17/miR-224 to increase the expression of $\mathrm{p} 21$ and PTEN to suppress $\mathrm{BC}$ progression (19). CircRNA circCEP128 functioned as a ceRNA for miR-145-5p and promoted progression of BC through increasing the expression of SOX11 (20). Several studies have reported the expression characteristics of circRNAs in BC tissues $(13,21)$. However, functional experiments are performed in BC cell lines and no studies have revealed the profile of circRNA expression using a microarray assay in BC cells. Thus, circRNA microarray analysis was carried out in SV-HUC-1, BC 5637 and T24 cells. The circRNA expression level in 5637 and T24 cells was compared with control SV-HUC-1. We found that circCASC15, was the most increased in 5637 and T24 cells, and it was also a upregulated significantly circRNA in a previous study using High-Throughout Sequencing (21). Highly expressed circCASC 15 was closely related to BC tumor stage. Upregulation of circCASC15 promoted cell proliferation in $\mathrm{BC}$ in vitro and in vivo. This is the first study to illustrate that circCASC 15 is clinically and functionally oncogenic in BC.

To investigate the underlying mechanism of circCASC15 in $B C$, we firstly illustrated the subcellular location of circCASC15 in BC cells through separating subcellular fractionation. circCASC15 was mainly localized in the cytoplasm, which was the same as miRNAs. We found that circCASC15 could bind to miR-1224-5p directly through bioinformatics analysis, RIP and dual-luciferase assays. Many studies have reported that miRNAs regulated gene expression through target mRNA degradation and inhibition of translation (22). In this study, circCASC15 could function as a miRNA sponges for miR-1224-5p and regulate the expression of the downstream gene CREB1. Many studies have shown that CREB1 played oncogenic roles to promote cell proliferation in different kinds of cancers including BC (23-27). Thus, we suspected that circCASC15 promoted cell proliferation through activating downstream CREB1 expression. Our results demonstrated that downregulation of circCASC15 could inhibit the mRNA and protein expression levels of CREB1 in BC 5637 and T24 cells. A rescue assay was performed, and the results of the CCK- 8 and EdU assays showed that overexpression of CREB1 could abrogate the effects of downregulation of cirCCASC15 on cell growth. 
In conclusion, the expression level of circCASC15 was positively correlated with tumor stage in $\mathrm{BC}$ patients, and one of the main mechanisms of circCASC15 was to act as a miR-1224-5p sponge to activate the oncogenic CREB1 expression to promote cell proliferation in BC. circCASC15 might be a novel clinical and therapeutic factor in $\mathrm{BC}$.

\section{MATERIALS AND METHODS}

\section{Patient tissue specimens}

Sixty-seven pairs of BC tissues and matched para-carcinoma tissues were acquired from patients who underwent radical cystectomy after being diagnosed with urothelial carcinomas of the bladder. This study was approved by the Institutional Ethical Committee Board of Peking University Shenzhen Hospital, and all patients signed informed consent before tissues collection. The 2004 World Health Organization Consensus Classification and Staging Systme for BC was used to classify specimens.

\section{Cell lines and cell culture}

Human BC (5637, T24, J82, UM-UC-3 and SW780) cell lines and the SV-HUC-1 cell line were purchased from American Type Culture Collection (ATCC, Manassas, VA, USA). Complete growth medium of these six cell lines was chosen according to ATCC suggestions. Cells were cultured at $37^{\circ} \mathrm{C}$ supplied with $5 \% \mathrm{CO}_{2}$ in an incubator.

\section{RNA extraction and quantitative real time-PCR (qRT-PCR)} TRIzol reagent (Invitrogen, Grand Island, NY, USA) was used to isolate total RNA from tissues or cell lines according to the manufacturer's instructions. Three U/mg RNase R was utilized to eliminate linear RNA at $37^{\circ} \mathrm{C}$ for $20 \mathrm{~min}$. The complementary DNA (cDNA) was synthesized from total RNA using a PrimeScript RT Reagent Kit with gDNA Eraser (Takara., Dalian, China) or a commerical miRNA reverse transcription PCR kit (RiboBio, Guangzhou, China). The SYBR Premix Ex TaqTM kit (Takara, Dalian, China) was used for the quantitative real-time PCR (qRT-PCR) analysis on the Roche lightcycler 480 Real-Time PCR System. Levels of GAPDH or U6 were used as an endogenous control to normalize the differences of circRNA and miRNA. The comparative $2^{-\Delta \Delta C T}$ method was used to analyze the expression levels. All assays were carried out at least three times. The primer sequences were described in Table S3.

\section{Additional methods}

Cell transfection, microarray analysis, vector construction, cell proliferation assays, subcellular fractionation location, RNAbinding protein immunoprecipitation (RIP) assay, dual-luciferase reporter assay, Western blot analysis and the xenograft tumor model are described in the Supplementary Materials and Methods.

\section{Statistical analysis}

All statistical analyses were carried out using SPSS computer software version 20.0 (SPSS Inc., Chicago, IL, USA). All data are indicated as the mean \pm standard error (SD). The data regarding circCASC15 expression level in BC tissues were analyzed using a paired-sample $t$-test. A Chi-square test was utilized to compare the difference in clinicopathological characteristics in BC patients. Student's t-test, was used to analyze the group difference. A P-value $<0.05$ (two-sided) was chosen for statistical significance.

\section{ACKNOWLEDGEMENTS}

This work was supported by grants from the Guangdong Key Laboratory of Male Reproductive Medicine and Genetics (2017B030314074), the Shenzhen Project of Science and Technology (JCYJ20170413100245260) and the 'San-ming' Project of Medicine in Shenzhen (SZSM201612066).

\section{CONFLICTS OF INTEREST}

The authors have no conflicting interests.

\section{REFERENCES}

1. Siegel RL, Miller KD and Jemal A (2017) Cancer Statistics, 2017. CA Cancer J Clin 67, 7-30

2. Cote RJ, Dunn MD, Chatterjee SJ et al (1998) Elevated and absent pRb expression is associated with bladder cancer progression and has cooperative effects with p53. Cancer Res 58, 1090-1094

3. Cambier S, Sylvester RJ, Collette L et al (2016) EORTC Nomograms and Risk Groups for Predicting Recurrence, Progression, and Disease-specific and Overall Survival in Non-Muscle-invasive Stage Ta-T1 Urothelial Bladder Cancer Patients Treated with 1-3 Years of Maintenance Bacillus Calmette-Guerin. Eur Urol 69, 60-69

4. Wu X, Liu D, Tao D et al (2016) BRD4 Regulates EZH2 Transcription through Upregulation of C-MYC and Represents a Novel Therapeutic Target in Bladder Cancer. Mol Cancer Ther 15, 1029-1042

5. Wilusz JE and Sharp PA (2013) Molecular biology. A circuitous route to noncoding RNA. Science 340, 440-441

6. Salzman J, Chen RE, Olsen MN, Wang PL and Brown PO (2013) Cell-type specific features of circular RNA expression. PLoS Genet 9, e1003777

7. Zhao ZJ and Shen J (2017) Circular RNA participates in the carcinogenesis and the malignant behavior of cancer. RNA Biol 14, 514-521

8. Hansen TB, Jensen TI, Clausen BH et al (2013) Natural RNA circles function as efficient microRNA sponges. Nature 495, 384-388

9. Liu H, Liu Y, Bian Z et al (2018) Circular RNA YAP1 inhibits the proliferation and invasion of gastric cancer cells by regulating the miR-367-5p/p27 (Kip1) axis. Mol Cancer 17, 151

10. Bai N, Peng E, Qiu X et al (2018) circFBLIM1 act as a 
ceRNA to promote hepatocellular cancer progression by sponging miR-346. J Exp Clin Cancer Res 37, 172

11. Su H, Tao T, Yang Z et al (2019) Circular RNA cTFRC acts as the sponge of MicroRNA-107 to promote bladder carcinoma progression. Mol Cancer 18, 27

12. Chen X, Chen RX, Wei WS et al (2018) PRMT5 Circular RNA Promotes Metastasis of Urothelial Carcinoma of the Bladder through Sponging miR-30c to Induce Epithelial-Mesenchymal Transition. Clin Cancer Res 24, 6319-6330

13. Li Y, Zheng F, Xiao X et al (2017) CircHIPK3 sponges miR-558 to suppress heparanase expression in bladder cancer cells. EMBO Rep 18, 1646-1659

14. Bolha L, Ravnik-Glavac M and Glavac D (2017) Circular RNAs: Biogenesis, Function, and a Role as Possible Cancer Biomarkers. Int J Genomics 2017, 6218353

15. Lewis BP, Burge CB and Bartel DP (2005) Conserved seed pairing, often flanked by adenosines, indicates that thousands of human genes are microRNA targets. Cell $120,15-20$

16. Zhong Z, Huang M, Lv M et al (2017) Circular RNA MYLK as a competing endogenous RNA promotes bladder cancer progression through modulating VEGFA/VEGFR2 signaling pathway. Cancer Lett 403, 305-317

17. Agarwal V, Bell GW, Nam JW and Bartel DP (2015) Predicting effective microRNA target sites in mammalian mRNAs. Elife 4, e05005

18. Xu Z, Yan Y, Zeng S et al (2018) Circular RNAs: clinical relevance in cancer. Oncotarget 9, 1444-1460

19. Yang C, Yuan W, Yang X et al (2018) Circular RNA circ-ITCH inhibits bladder cancer progression by sponging miR-17/miR-224 and regulating p21, PTEN expression.
Mol Cancer 17, 19

20. Wu Z, Huang W, Wang $X$ et al (2018) Circular RNA CEP128 acts as a sponge of miR-145-5p in promoting the bladder cancer progression via regulating SOX11. Mol Med 24, 40

21. Yang X, Yuan W, Tao J et al (2017) Identification of circular RNA signature in bladder cancer. J Cancer 8, 3456-3463

22. Imani S, Wu RC and Fu J (2018) MicroRNA-34 family in breast cancer: from research to therapeutic potential. J Cancer 9, 3765-3775

23. Gu L, Lu LS, Zhou DL and Liu ZC (2018) UCA1 promotes cell proliferation and invasion of gastric cancer by targeting CREB1 sponging to miR-590-3p. Cancer Med 7, 1253-1263

24. Sunkel B, Wu D, Chen Z et al (2017) Integrative analysis identifies targetable CREB1/FoxA1 transcriptional co-regulation as a predictor of prostate cancer recurrence. Nucleic Acids Res 45, 6993

25. Pan J, Li X, Wu W et al (2016) Long non-coding RNA UCA1 promotes cisplatin/gemcitabine resistance through CREB modulating miR-196a-5p in bladder cancer cells. Cancer Lett 382, 64-76

26. Xu X, Zhu Y, Liang Z et al (2016) c-Met and CREB1 are involved in miR-433-mediated inhibition of the epithelial-mesenchymal transition in bladder cancer by regulating Akt/GSK-3beta/Snail signaling. Cell Death Dis 7, e2088

27. Yang C, Li X, Wang Y, Zhao L and Chen W (2012) Long non-coding RNA UCA1 regulated cell cycle distribution via CREB through PI3-K dependent pathway in bladder carcinoma cells. Gene 496, 8-16 